\title{
Impulsive Multiorders Riemann-Liouville Fractional Differential Equations
}

\author{
Weera Yukunthorn, ${ }^{1,2}$ Sotiris K. Ntouyas, ${ }^{3,4}$ and Jessada Tariboon ${ }^{1,2}$ \\ ${ }^{1}$ Nonlinear Dynamic Analysis Research Center, Department of Mathematics, Faculty of Applied Science, \\ King Mongkut's University of Technology North Bangkok, Bangkok 10800, Thailand \\ ${ }^{2}$ Centre of Excellence in Mathematics, CHE, Si Ayutthaya Road, Bangkok 10400, Thailand \\ ${ }^{3}$ Department of Mathematics, University of Ioannina, 45110 Ioannina, Greece \\ ${ }^{4}$ Nonlinear Analysis and Applied Mathematics (NAAM) Research Group, Department of Mathematics, Faculty of Science, \\ King Abdulaziz University, P.O. Box 80203, Jeddah 21589, Saudi Arabia
}

Correspondence should be addressed to Jessada Tariboon; jessadat@kmutnb.ac.th

Received 9 October 2014; Accepted 6 April 2015

Academic Editor: Seenith Sivasundaram

Copyright (C) 2015 Weera Yukunthorn et al. This is an open access article distributed under the Creative Commons Attribution License, which permits unrestricted use, distribution, and reproduction in any medium, provided the original work is properly cited.

Impulsive multiorders fractional differential equations are studied. Existence and uniqueness results are obtained for first- and second-order impulsive initial value problems by using Banach's fixed point theorem in an appropriate weighted space. Examples illustrating the main results are presented.

\section{Introduction}

Fractional calculus has become very useful over the last years because of its many applications in almost all applied sciences. By now, almost all fields of research in science and engineering use fractional calculus to better describe them.

Fractional differential equations have been of great interest and are caused both by the intensive development of the theory of fractional calculus itself and by the applications of such constructions in various science such as physics, mechanics, chemistry, and engineering. For details and some recent results on the subject we refer to the papers [1-3], books [4-7], and references cited therein.

Recently in [8], Wang et al. studied existence and uniqueness results for the following impulsive multipoint fractional integral boundary value problem involving multiorders fractional derivatives and deviating argument:

$$
\begin{aligned}
{ }^{c} D_{t_{k}^{+}}^{\alpha_{k}} u(t) & =f(t, u(t), u(\theta(t))), \quad 1<\alpha_{k} \leq 2, \\
\Delta u\left(t_{k}\right) & =I_{k}\left(u\left(t_{k}\right)\right), \\
\Delta u^{\prime}\left(t_{k}\right) & =I_{k}^{*}\left(u\left(t_{k}\right)\right),
\end{aligned}
$$

$$
\begin{aligned}
u(0) & =\sum_{k=0}^{p} \lambda_{k} \mathscr{F}_{t_{k}^{+}}^{\beta_{k}} u\left(\eta_{k}\right), \\
u^{\prime}(0) & =0,
\end{aligned}
$$

$$
t_{k}<\eta_{k}<t_{k+1}
$$

where ${ }^{c} D_{t_{k}^{+}}^{\alpha_{k}}$ is the Caputo fractional derivative of order $\alpha_{k}, \mathscr{J}_{t_{k}^{+}}^{\beta_{k}}$ is fractional Riemann-Liouville integral of order $\beta_{k}>0, f^{k_{k}} \in$ $C(J \times \mathbb{R} \times \mathbb{R}, \mathbb{R}), I_{k}, I_{k}^{*} \in C(\mathbb{R}, \mathbb{R}), \theta \in C(J, J), J=[0, T](T>$ $0), 0=t_{0}<t_{1}<\cdots<t_{k}<\cdots<t_{p}<t_{p+1}=T, \Delta u\left(t_{k}\right)=$ $u\left(t_{k}^{+}\right)-u\left(t_{k}^{-}\right)$, and $\Delta u^{\prime}\left(t_{k}\right)=u^{\prime}\left(t_{k}^{+}\right)-u^{\prime}\left(t_{k}^{-}\right)$where $u\left(t_{k}^{+}\right), u^{\prime}\left(t_{k}^{+}\right)$ and $u\left(t_{k}^{-}\right), u^{\prime}\left(t_{k}^{-}\right)$denote the right and left hand limits of $u(t)$ and $u^{\prime}(t)$ at $t=t_{k}(k=1,2, \ldots, p)$.

We notice that there are some discussions on the concept of solution for impulsive fractional differential equations for both Riemann-Liouville and Caputo fractional derivatives. We refer the interested reader to some recent papers [911] and the references cited therein. However, we can point out the problems caused by using the definition of Caputo 
fractional derivatives of order $\alpha$ with the lower limit 0 for a function $f$ as

$$
{ }^{c} D_{0}^{\alpha} f(t)=\frac{1}{\Gamma(n-\alpha)} \int_{0}^{t}(t-s)^{n-\alpha-1} f^{(n)}(s) d s .
$$

If there are impulse points $t_{k}$ such that $t_{k} \in(0, t)$ for some $k \in \mathbb{N}$, then the $f^{(n)}\left(t_{k}\right)$ does not exist, which leads to nonintegrability of the right-hand side of (2). The key idea for solving this problem is to apply the definition of fractional derivative only on an interval $\left(t_{k}, t_{k+1}\right)$ and combining all intervals by using impulsive conditions.

In this paper, we study impulsive multiorders RiemannLiouville fractional differential equations. More precisely, in Section 3 we study the existence and uniqueness of solutions for the following initial value problem for impulsive multiorders Riemann-Liouville fractional differential equations of order $0<\alpha_{k} \leq 1$ of the form

$$
\begin{aligned}
D_{t_{k}}^{\alpha_{k}} x(t) & =f(t, x(t)), \quad t \in J, t \neq t_{k}, \\
\widetilde{\Delta} x\left(t_{k}\right) & =\varphi_{k}\left(x\left(t_{k}\right)\right), \quad k=1,2,3, \ldots, m, \\
x(0) & =0,
\end{aligned}
$$

where $D_{t_{k}}^{\alpha_{k}}$ is the Riemann-Liouville fractional derivative of order $0<\alpha_{k} \leq 1$ on intervals $J_{k}, k=0,1,2, \ldots, m, 0=t_{0}<$ $t_{1}<t_{2}<\cdots<t_{k}<\cdots<t_{m}<t_{m+1}=T, f: J \times \mathbb{R} \rightarrow \mathbb{R}$ is a continuous function, and $\varphi_{k} \in C(\mathbb{R}, \mathbb{R})$. The notation $\widetilde{\Delta} x\left(t_{k}\right)$ is defined by

$$
\begin{aligned}
\widetilde{\Delta} x\left(t_{k}\right)=I_{t_{k}}^{1-\alpha_{k}} x\left(t_{k}^{+}\right)-I_{t_{k-1}}^{1-\alpha_{k-1}} x\left(t_{k}\right), & \\
& k=1,2,3, \ldots, m,
\end{aligned}
$$

where $I_{t_{k}}^{1-\alpha_{k}}$ is the Riemann-Liouville fractional integral of order $1-\alpha_{k}$ on interval $J_{k}$. It should be noticed that if $\alpha_{k}=$ 1 in (4), then $\widetilde{\Delta} x\left(t_{k}\right)=\Delta x\left(t_{k}\right)=x\left(t_{k}^{+}\right)-x\left(t_{k}\right)$ for $k=$ $1,2,3, \ldots, m$.

In Section 4, we investigate the initial value problem of impulsive Riemann-Liouville fractional differential equations of the form

$$
\begin{aligned}
D_{t_{k}}^{\alpha_{k}} x(t) & =f(t, x(t)), \quad t \in J, t \neq t_{k}, \\
\widetilde{\Delta} x\left(t_{k}\right) & =\varphi_{k}\left(x\left(t_{k}\right)\right), \quad k=1,2,3, \ldots, m, \\
\Delta^{*} x\left(t_{k}\right) & =\varphi_{k}^{*}\left(x\left(t_{k}\right)\right), \quad k=1,2,3, \ldots, m, \\
x(0) & =0, \\
D^{\alpha_{0}-1} x(0) & =\beta,
\end{aligned}
$$

where $\beta \in \mathbb{R}, 0=t_{0}<t_{1}<t_{2}<\cdots<t_{k}<\cdots<t_{m}<$ $t_{m+1}=T, f: J \times \mathbb{R} \rightarrow \mathbb{R}$ is a continuous function, $\varphi_{k}, \varphi_{k}^{*} \in$ $C(\mathbb{R}, \mathbb{R})$ for $k=1,2, \ldots, m$, and $D_{t_{k}}^{\alpha_{k}}$ is the Riemann-Liouville fractional derivative of order $1<\alpha_{k} \leq 2$ on intervals $J_{k}$ for $k=0,1,2, \ldots, m$. The notation $\widetilde{\Delta} x\left(t_{k}\right)$ is defined by (4) and $\Delta^{*} x\left(t_{k}\right)$ is defined by

$$
\Delta^{*} x\left(t_{k}\right)=I_{t_{k}}^{2-\alpha_{k}} x\left(t_{k}^{+}\right)-I_{t_{k-1}}^{2-\alpha_{k-1}} x\left(t_{k}\right),
$$

where $I_{t_{k}}^{2-\alpha_{k}}$ is the Riemann-Liouville fractional integral of order $2-\alpha_{k}>0$ on $J_{k}$. It should be noticed that if $\alpha_{k}=2$ in (6), then $\widetilde{\Delta} x\left(t_{k}\right)=D_{t_{k}} x\left(t_{k}^{+}\right)-D_{t_{k-1}} x\left(t_{k}\right)$ and $\Delta^{*} x\left(t_{k}\right)=$ $\Delta x\left(t_{k}\right)=x\left(t_{k}^{+}\right)-x\left(t_{k}\right)$ for $k=1,2, \ldots, m$.

By using Banach's fixed point theorem we prove existence and uniqueness results for the problem (3) and (5) in an appropriate weighted space.

The paper is organized as follows: Section 2 contains some preliminary notations, definitions, and lemmas that we need in the sequel. In Section 3 we present the main results for problem (3), while in Section 4 we present the main results for problem (5). Examples illustrating the obtained results are also presented.

\section{Preliminaries}

In this section, we introduce some notations and definitions of fractional calculus and present preliminary results needed in our proofs later.

Definition 1. The Riemann-Liouville fractional derivative of order $\alpha>0$ of a continuous function $f:(a, b) \rightarrow \mathbb{R}$ is defined by

$$
\begin{aligned}
D_{a}^{\alpha} f(t)=\frac{1}{\Gamma(n-\alpha)}\left(\frac{d}{d t}\right)^{n} \int_{a}^{t}(t-s)^{n-\alpha-1} f(s) d s, \\
n-1<\alpha<n, t \in(a, b),
\end{aligned}
$$

where $n=[\alpha]+1,[\alpha]$ denotes the integer part of a real number $\alpha$, provided the right-hand side is pointwise defined on $(a, b)$, where $\Gamma$ is the gamma function defined by $\Gamma(\alpha)=$ $\int_{0}^{\infty} e^{-s} s^{\alpha-1} d s$.

Definition 2. For at least $n$-times differentiable function $f$ : $(a, b) \rightarrow \mathbb{R}$, the Caputo derivative of fractional order $\alpha$ is defined as

$$
\begin{array}{r}
{ }^{c} D_{a}^{\alpha} f(t)=\frac{1}{\Gamma(n-\alpha)} \int_{a}^{t}(t-s)^{n-\alpha-1} f^{(n)}(s) d s, \\
n-1<\alpha<n, t \in(a, b),
\end{array}
$$

where $n=[\alpha]+1$.

Definition 3. The Riemann-Liouville fractional integral of order $\beta>0$ of a continuous function $f:(a, b) \rightarrow \mathbb{R}$ is defined by

$$
I_{a}^{\alpha} f(t)=\frac{1}{\Gamma(\alpha)} \int_{a}^{t}(t-s)^{\alpha-1} f(s) d s, \quad t \in(a, b)
$$

provided the right-hand side is pointwise defined on $(a, b)$.

Lemma 4 (see [5]). Let $\alpha>0$ and $x \in C(a, b) \cap L(a, b)$. Then the fractional differential equation

$$
D_{a}^{\alpha} x(t)=0
$$

has a unique solution

$$
\begin{aligned}
x(t)= & k_{1}(t-a)^{\alpha-1}+k_{2}(t-a)^{\alpha-2}+\cdots \\
& +k_{n}(t-a)^{\alpha-n},
\end{aligned}
$$

where $k_{i} \in \mathbb{R}, i=1,2, \ldots, n$, and $n-1<q<n$.

$$
k=1,2, \ldots, m,
$$


Lemma 5 (see [5]). Let $\alpha>0$. Then for $x \in C(a, b) \cap L(a, b)$ it holds

$$
I_{a}^{\alpha} D_{a}^{\alpha} x(t)=x(t)-\sum_{j=1}^{n} \frac{\left(I_{a}^{n-\alpha} x\right)^{(n-j)}(a)}{\Gamma(\alpha-j+1)}(t-a)^{\alpha-j},
$$

where $n-1<\alpha<n$.

\section{Impulsive Riemann-Liouville Fractional Differential Equations of Orders $0<\alpha_{k} \leq 1$}

Let $J=[0, T], J_{0}=\left[t_{0}, t_{1}\right], J_{k}=\left(t_{k}, t_{k+1}\right]$ for $k=1,2,3, \ldots, m$. Let $P C(J, \mathbb{R})=\{x: J \rightarrow \mathbb{R}, x(t)$ is continuous everywhere except for some $t_{k}$ at which $x\left(t_{k}^{+}\right)$and $x\left(t_{k}^{-}\right)$exist, and $x\left(t_{k}^{-}\right)=$ $\left.x\left(t_{k}\right), k=1,2,3, \ldots, m\right\}$. For $\gamma \in \mathbb{R}_{+}$, we introduce the space $C_{\gamma, k}\left(J_{k}, \mathbb{R}\right)=\left\{x: J_{k} \rightarrow \mathbb{R}:\left(t-t_{k}\right)^{\gamma} x(t) \in C\left(J_{k}, \mathbb{R}\right)\right\}$ with the norm $\|x\|_{C_{\gamma, k}}=\sup _{t \in J_{k}}\left|\left(t-t_{k}\right)^{\gamma} x(t)\right|$ and $P C_{\gamma}=\{x: J \rightarrow \mathbb{R}:$ for each $t \in J_{k}$ and $\left.\left(t-t_{k}\right)^{\gamma} x(t) \in C\left(J_{k}, \mathbb{R}\right), k=0,1,2, \ldots, m\right\}$ with the norm $\|x\|_{P C_{y}}=\max \left\{\sup _{t \in J_{k}}\left|\left(t-t_{k}\right)^{\gamma} x(t)\right|: k=\right.$ $0,1,2, \ldots, m\}$. Clearly $P C_{\gamma}$ is a Banach space.

In this section we study problem (3).

Lemma 6. If $x \in P C(J, \mathbb{R})$ is a solution of (3), then, for any $t \in J_{k}, k=0,1,2, \ldots, m$,

$$
\begin{gathered}
x(t)=\frac{\left(t-t_{k}\right)^{\alpha_{k}-1}}{\Gamma\left(\alpha_{k}\right)}\left[\sum _ { 0 < t _ { k } < t } \left(I_{t_{k-1}}^{1} f\left(t_{k}, x\left(t_{k}\right)\right)\right.\right. \\
\left.\left.+\varphi_{k}\left(x\left(t_{k}\right)\right)\right)\right]+I_{t_{k}}^{\alpha_{k}} f(t, x(t)),
\end{gathered}
$$

with $\sum_{0<0}(\cdot)=0$. The converse is also true.

Proof. For $t \in J_{0}$, taking the Riemann-Liouville fractional integral of order $\alpha_{0}$ to the first equation of (3) and using Lemma 5, we have

$$
x(t)=I_{t_{0}}^{\alpha_{0}} f(t, x(t))+c_{0} \frac{t^{\alpha_{0}-1}}{\Gamma\left(\alpha_{0}\right)},
$$

where $c_{0}=I_{t_{0}}^{1-\alpha_{0}} x(0)$. The initial condition $x(0)=0$ implies $c_{0}=0$. Then for $x \in J_{0}$, we get

$$
x(t)=I_{t_{0}}^{\alpha_{0}} f(t, x(t)) .
$$

Applying the Riemann-Liouville fractional integral of order $1-\alpha_{0}$ from 0 to $t_{1}$, we get

$$
I_{t_{0}}^{1-\alpha_{0}} x\left(t_{1}\right)=I_{t_{0}}^{1-\alpha_{0}} I_{t_{0}}^{\alpha_{0}} f\left(t_{1}, x\left(t_{1}\right)\right)=I_{t_{0}}^{1} f\left(t_{1}, x\left(t_{1}\right)\right) .
$$

For $t \in J_{1}$, taking the Riemann-Liouville fractional integral of order $\alpha_{1}$ to the first equation of (3) and using Lemma 5, we have

$$
x(t)=\frac{\left(t-t_{1}\right)^{\alpha_{1}-1}}{\Gamma\left(\alpha_{1}\right)} I_{t_{1}}^{1-\alpha_{1}} x\left(t_{1}^{+}\right)+I_{t_{1}}^{\alpha_{1}} f(t, x(t)) .
$$

Since $I_{t_{1}}^{1-\alpha_{1}} x\left(t_{1}^{+}\right)=I_{t_{0}}^{1-\alpha_{0}} x\left(t_{1}\right)+\varphi_{1}\left(x\left(t_{1}\right)\right)$, it follows for $t \in J_{1}$ that

$$
\begin{aligned}
x(t)= & \frac{\left(t-t_{1}\right)^{\alpha_{1}-1}}{\Gamma\left(\alpha_{1}\right)}\left[I_{t_{0}}^{1} f\left(t_{1}, x\left(t_{1}\right)\right)+\varphi_{1}\left(x\left(t_{1}\right)\right)\right] \\
& +I_{t_{1}}^{\alpha_{1}} f(t, x(t)) .
\end{aligned}
$$

Applying the Riemann-Liouville fractional integral of order $1-\alpha_{1}$ to the above equation and substituting $t=t_{1}$, one has

$$
\begin{aligned}
I_{t_{1}}^{1-\alpha_{1}} x(t)= & I_{t_{0}}^{1} f\left(t_{1}, x\left(t_{1}\right)\right)+\varphi_{1}\left(x\left(t_{1}\right)\right) \\
& +I_{t_{1}}^{1} f(t, x(t)) .
\end{aligned}
$$

For $t \in J_{2}$, using the Riemann-Liouville fractional integral of order $\alpha_{2}$ for (3), we have

$$
\begin{aligned}
x(t) & =\frac{\left(t-t_{2}\right)^{\alpha_{2}-1}}{\Gamma\left(\alpha_{2}\right)} I_{t_{2}}^{1-\alpha_{2}} x\left(t_{2}^{+}\right)+I_{t_{2}}^{\alpha_{2}} f(t, x(t)) \\
= & \frac{\left(t-t_{2}\right)^{\alpha_{2}-1}}{\Gamma\left(\alpha_{2}\right)}\left[I_{t_{1}}^{1-\alpha_{1}} x\left(t_{2}\right)+\varphi_{2}\left(x\left(t_{2}\right)\right)\right] \\
& +I_{t_{2}}^{\alpha_{2}} f(t, x(t))=\frac{\left(t-t_{2}\right)^{\alpha_{2}-1}}{\Gamma\left(\alpha_{2}\right)}\left[I_{t_{0}}^{1} f\left(t_{1}, x\left(t_{1}\right)\right)\right. \\
& \left.+\varphi_{1}\left(x\left(t_{1}\right)\right)+I_{t_{1}}^{1} f\left(t_{2}, x\left(t_{2}\right)\right)+\varphi_{2}\left(x\left(t_{2}\right)\right)\right] \\
& +I_{t_{2}}^{\alpha_{2}} f(t, x(t)) .
\end{aligned}
$$

Repeating the above procession for each $J_{k}$, we obtain (13).

On the other hand, assume that $x$ is a solution of (3). Applying the Riemann-Liouville fractional derivative of order $\alpha_{k}$ for (13) on $J_{k}, k=0,1,2, \ldots, m$, and using $\Gamma(0)=\infty$, it follows that

$$
D_{t_{k}}^{\alpha_{k}} x(t)=f(t, x(t)) .
$$

It is easy to verify that $\widetilde{\Delta} x\left(t_{k}\right)=\varphi_{k}\left(x\left(t_{k}\right)\right), k=1,2,3, \ldots, m$, and $x(0)=0$. The proof is complete.

Next we will prove that problem (3) has a unique solution by using Banach's fixed point theorem.

Theorem 7. Assume that

$\left(H_{1}\right) f: J \times \mathbb{R} \rightarrow \mathbb{R}$ is a continuous function and satisfies

$|f(t, x)-f(t, y)| \leq L_{1}|x-y|$,

$$
L_{1}>0, \forall t \in J, x, y \in \mathbb{R}
$$

$\left(H_{2}\right) \varphi_{k}: \mathbb{R} \rightarrow \mathbb{R}, k=1,2,3, \ldots, m$, are continuous functions and satisfy

$$
\left|\varphi_{k}(x)-\varphi_{k}(y)\right| \leq L_{2}|x-y|, \quad L_{2}>0, \forall x, y \in \mathbb{R} .
$$

If

$$
\Lambda_{1}:=\frac{T^{*}}{\Gamma^{*}}\left(L_{1}+L_{1} T+L_{2} m\right)<1,
$$

where $T^{*}=\max \left\{T^{\gamma+\alpha_{k}-1}, T^{\gamma+\alpha_{k}}\right\}, \Gamma^{*}=\min \left\{\Gamma\left(\alpha_{k}\right), \Gamma\left(\alpha_{k}+1\right)\right\}$, and $\gamma+\alpha_{k}>1$ for $k=0,1,2, \ldots, m$, then the initial value problem (3) has a unique solution on $[0, T]$. 
Proof. In view of Lemma 6, we define the operator $\mathscr{K}:$ $P C \rightarrow P C$ as

$$
\begin{aligned}
& \mathscr{K} x(t)=\frac{\left(t-t_{k}\right)^{\alpha_{k}-1}}{\Gamma\left(\alpha_{k}\right)}\left[\sum _ { 0 < t _ { k } < t } \left(I_{t_{k-1}}^{1} f\left(t_{k}, x\left(t_{k}\right)\right)\right.\right. \\
& \left.\left.+\varphi_{k}\left(x\left(t_{k}\right)\right)\right)\right]+I_{t_{k}}^{\alpha_{k}} f(t, x(t)) .
\end{aligned}
$$

In addition, we define a ball $B_{r}=\left\{x \in P C_{\gamma}(J, \mathbb{R}),\|x\|_{P C_{\gamma}} \leq r\right\}$. To show that $\mathscr{K} x \in P C_{\gamma}$, we suppose $\tau \in J_{k}$ and then

$$
\begin{aligned}
& \left|\left(t-t_{k}\right)^{\gamma} \mathscr{K} x(t)-\left(\tau-t_{k}\right)^{\gamma} \mathscr{K} x(\tau)\right| \\
& \leq \mid \frac{\left(t-t_{k}\right)^{\gamma+\alpha_{k}-1}}{\Gamma\left(\alpha_{k}\right)}\left[\sum_{j=1}^{k}\left(I_{t_{j-1}}^{1} f\left(t_{j}, x\left(t_{j}\right)\right)+\varphi_{j}\left(x\left(t_{j}\right)\right)\right)\right] \\
& \quad+\left(t-t_{k}\right)^{\gamma} I_{t_{k}}^{\alpha_{k}} f(t, x(t)) \\
& \quad-\frac{\left(\tau-t_{k}\right)^{\gamma+\alpha_{k}-1}}{\Gamma\left(\alpha_{k}\right)}\left[\sum_{j=1}^{k}\left(I_{t_{j-1}}^{1} f\left(t_{j}, x\left(t_{j}\right)\right)+\varphi_{j}\left(x\left(t_{j}\right)\right)\right)\right] \\
& \quad-\left(\tau-t_{k}\right)^{\gamma} I_{t_{k}}^{\alpha_{k}} f(\tau, x(\tau)) \mid \\
& \quad \leq\left|\frac{\left(t-t_{k}\right)^{\gamma+\alpha_{k}-1}-\left(\tau-t_{k}\right)^{\gamma+\alpha_{k}-1}}{\Gamma\left(\alpha_{k}\right)}\right| \mid \sum_{j=1}^{k}\left(I_{t_{j-1}}^{1} f\left(t_{j}, x\left(t_{j}\right)\right)\right. \\
& \left.\quad+\varphi_{j}\left(x\left(t_{j}\right)\right)\right)|+|\left(t-t_{k}\right)^{\gamma} I_{t_{k}}^{\alpha_{k}} f(t, x(t))-\left(\tau-t_{k}\right)^{\gamma} \\
& \quad \cdot I_{t_{k}}^{\alpha_{k}} f(\tau, x(\tau)) \mid .
\end{aligned}
$$

As $t \rightarrow \tau$, we get $\left|\left(t-t_{k}\right)^{\gamma} \mathscr{K} x(t)-\left(\tau-t_{k}\right)^{\gamma} \mathscr{K} x(\tau)\right| \rightarrow 0$ for each $k=0,1,2, \ldots, m$. Therefore, we have $\mathscr{K} x(t) \in$ $P C_{\gamma}$. Next we will show that $\mathscr{K} B_{r} \subset B_{r}$. Suppose that $\sup _{t \in J}|f(t, 0)|=M, \max \left\{\left|I_{k}(0)\right|, k=1,2,3, \ldots, m\right\}=N$. Setting

$$
\Lambda_{2}=\frac{T^{*}}{\Gamma^{*}}(M+M T+N m)
$$

we choose $r$ such that

$$
r \geq \frac{\Lambda_{2}}{1-\Lambda_{1}}
$$

For any $x \in B_{r}$ and for each $t \in J_{k}$, we have

$$
\begin{aligned}
& |(\mathscr{K} x)(t)| \leq \frac{\left(t-t_{k}\right)^{\alpha_{k}-1}}{\Gamma\left(\alpha_{k}\right)}\left[\sum _ { j = 1 } ^ { k } \left(I_{t_{j-1}}^{1}|f(s, x(s))|\left(t_{j}\right)\right.\right. \\
& \left.\left.+\left|\varphi_{j}\left(x\left(t_{j}\right)\right)\right|\right)\right]+I_{t_{k}}^{\alpha_{k}}|f(s, x(s))|(t) \\
& \quad \leq \frac{\left(t-t_{k}\right)^{\alpha_{k}-1}}{\Gamma\left(\alpha_{k}\right)}\left[\sum _ { j = 1 } ^ { k } \left(I_{t_{j-1}}^{1}(|f(s, x(s))-f(s, 0)|+|f(s, 0)|)\right.\right.
\end{aligned}
$$

$$
\begin{aligned}
& \left.\left.\cdot\left(t_{j}\right)+\left|\varphi_{j}\left(x\left(t_{j}\right)\right)-\varphi_{j}(0)\right|+\left|\varphi_{j}(0)\right|\right)\right]+I_{t_{k}}^{\alpha_{k}}(\mid f(s, x(s)) \\
& -f(s, 0)|-| f(s, 0) \mid)(t) \leq \frac{\left(t-t_{k}\right)^{\alpha_{k}-1}}{\Gamma\left(\alpha_{k}\right)}\left[\left(L_{1} r+M\right) t_{k}\right. \\
& \left.+\left(L_{2} r+N\right) k\right]+\frac{\left(t-t_{k}\right)^{\alpha_{k}}}{\Gamma\left(\alpha_{k}+1\right)}\left(L_{1} r+M\right) .
\end{aligned}
$$

Multiplying both sides of the above inequality by $\left(t-t_{k}\right)^{\gamma}$ for each $t \in J_{k}$, we obtain

$$
\begin{aligned}
\left(t-t_{k}\right)^{\gamma}|(\mathscr{K} x)(t)| & \\
\leq & \frac{\left(t-t_{k}\right)^{\gamma+\alpha_{k}-1}}{\Gamma\left(\alpha_{k}\right)}\left[\left(L_{1} r+M\right) t_{k}+\left(L_{2} r+N\right) k\right] \\
& +\frac{\left(t-t_{k}\right)^{\gamma+\alpha_{k}}}{\Gamma\left(\alpha_{k}+1\right)}\left(L_{1} r+M\right) \\
\leq & r\left(\frac{T^{*}}{\Gamma^{*}}\left(L_{1}+L_{1} T+L_{2} m\right)\right) \\
& +\left(\frac{T^{*}}{\Gamma^{*}}(M+M T+N m)\right)=r \Lambda_{1}+\Lambda_{2} \leq r .
\end{aligned}
$$

This implies that $\mathscr{K} B_{r} \subset B_{r}$.

Finally we will show that $\mathscr{K}$ is a contraction mapping on $B_{r}$. For $x, y \in P C_{\gamma}(J, \mathbb{R})$ and for each $t \in J$ we have

$$
\begin{aligned}
& |(\mathscr{K} x)(t)-(\mathscr{K} y)(t)| \\
& \leq \frac{\left(t-t_{k}\right)^{\alpha_{k}-1}}{\Gamma\left(\alpha_{k}\right)}\left[\sum _ { j = 1 } ^ { k } \left(I_{t_{j-1}}^{1}|f(s, x(s))-f(s, y(s))|\right.\right. \\
& \left.\left.\cdot\left(t_{j}\right)+\left|\varphi_{j}\left(x\left(t_{j}\right)\right)-\varphi_{j}\left(y\left(t_{j}\right)\right)\right|\right)\right] \\
& +I_{t_{k}}^{\alpha_{k}}|f(s, x(s))-f(s, y(s))|(t) \\
& \leq \frac{\left(t-t_{k}\right)^{\alpha_{k}-1}}{\Gamma\left(\alpha_{k}\right)}\left[\sum _ { j = 1 } ^ { k } \left(L_{1}\left(t_{j}-t_{j-1}\right)\|x-y\|\right.\right. \\
& \left.\left.+L_{2}\|x-y\|\right)\right]+\frac{\left(t-t_{k}\right)^{\alpha_{k}}}{\Gamma\left(\alpha_{k}+1\right)} L_{1}\|x-y\| .
\end{aligned}
$$

Multiplying both sides of the above inequality by $\left(t-t_{k}\right)^{\gamma}$ for each $t \in J_{k}$, we have

$$
\begin{aligned}
& \left|\left(t-t_{k}\right)^{\gamma}(\mathscr{K} x)(t)-\left(t-t_{k}\right)^{\gamma}(\mathscr{K} y)(t)\right| \\
& \quad \leq \frac{\left(t-t_{k}\right)^{\gamma+\alpha_{k}-1}}{\Gamma\left(\alpha_{k}\right)}\left(L_{1} t_{k}\|x-y\|+L_{2} k\|x-y\|\right)
\end{aligned}
$$




$$
\begin{aligned}
& +\frac{\left(t-t_{k}\right)^{\gamma+\alpha_{k}}}{\Gamma\left(\alpha_{k}+1\right)} L_{1}\|x-y\| \\
\leq & \frac{T^{*}}{\Gamma^{*}}\left(L_{1}+L_{1} T+L_{2} m\right)\|x-y\| .
\end{aligned}
$$

It follows that

$$
\|\mathscr{K} x-\mathscr{K} y\| \leq \Lambda_{1}\|x-y\| .
$$

Since $\Lambda_{1}<1, \mathscr{K}$ is a contraction mapping on $B_{r}$. Therefore (3) has a unique solution on $[0, T]$.

Example 8. Consider the following impulsive multiorders Riemann-Liouville fractional initial value problem:

$$
\begin{aligned}
D_{t_{k}}^{(k+1) /(k+2)} x & =\frac{|x(t)| \ln (t+1)^{2}}{(4 t+3)^{3}(1+2|x(t)|)}+\frac{e^{t} \cos t}{5-2 t}, \\
t \in\left[0, \frac{11}{10}\right], t \neq t_{k}, & \\
\widetilde{\Delta} x\left(t_{k}\right) & =\frac{\left|x\left(t_{k}\right)\right|}{(4 k) !+\left|x\left(t_{k}\right)\right|}, \\
k & =1,2, \ldots, 10, t_{k}=\frac{k}{10},
\end{aligned}
$$

$$
x(0)=0 .
$$

Here $\alpha_{k}=(k+1) /(k+2), k=0,1,2, \ldots, 10, m=10$, $T=11 / 10, f(t, x)=\left(|x| \ln (t+1)^{2}\right) /\left((4 t+3)^{3}(1+2|x|)\right)+$ $\left(e^{t} \cos t\right) /(5-2 t)$, and $\varphi_{k}(x)=|x| /((4 k) !+|x|)$. Since $\mid f(t, x)-$ $f(t, y)|\leq(2 / 27)| x-y \mid$ and $\left|\varphi_{k}(x)-\varphi_{k}(y)\right| \leq(1 / 24)|x-y|$, for $k=1,2, \ldots, 10$, then $\left(H_{1}\right)$ and $\left(H_{2}\right)$ are satisfied with $L_{1}=$ $2 / 27, L_{2}=1 / 24$.

By choosing $\gamma=1$, we find that $T^{*}=1.200428, \Gamma^{*}=$ 0.886227 , and

$$
\Lambda_{1}=\frac{T^{*}}{\Gamma^{*}}\left(L_{1}+L_{1} T+L_{2} m\right)=0.775096<1 .
$$

Hence, by Theorem 7, the initial value problem (34) has a unique solution on $[0,11 / 10]$.

\section{Impulsive Riemann-Liouville Fractional Differential Equations of Orders $1<\alpha_{k} \leq 2$}

Problem (5) is studied in this section.

Lemma 9. The unique solution of problem (5) is given by

$$
\begin{aligned}
x(t) & =\frac{\left(t-t_{k}\right)^{\alpha_{k}-2}}{\Gamma\left(\alpha_{k}-1\right)}\left[\beta t_{k}\right. \\
& +\sum_{j=1}^{k-1}\left(t_{k}-t_{j}\right)\left(I_{t_{j-1}}^{1} f\left(t_{j}, x\left(t_{j}\right)\right)+\varphi_{j}\left(x\left(t_{j}\right)\right)\right)
\end{aligned}
$$

$$
\begin{aligned}
& \left.+\sum_{j=1}^{k}\left(I_{t_{j-1}}^{2} f\left(t_{j}, x\left(t_{j}\right)\right)+\varphi_{j}^{*}\left(x\left(t_{j}\right)\right)\right)\right] \\
& +\frac{\left(t-t_{k}\right)^{\alpha_{k}-1}}{\Gamma\left(\alpha_{k}\right)}[\beta
\end{aligned}
$$$$
\left.+\sum_{j=1}^{k}\left(I_{t_{j-1}}^{1} f\left(t_{j}, x\left(t_{j}\right)\right)+\varphi_{j} x\left(t_{j}\right)\right)\right]+I_{t_{k}}^{\alpha_{k}} f(t,
$$$$
x(t)) \text {, }
$$

for $t \in J_{k}, k=0,1,2, \ldots, m$ with $\sum_{j=a}^{b}=0$ for $b<a$.

Proof. For $t \in J_{0}$, taking the Riemann-Liouville fractional integral of order $\alpha_{0}$ for the first equation of (5) and applying Lemma 5, we obtain

$$
x(t)=I_{t_{0}}^{\alpha_{0}} f(t, x(t))+c_{0} \frac{t^{\alpha_{0}-1}}{\Gamma\left(\alpha_{0}\right)}+c_{1} \frac{t^{\alpha_{0}-2}}{\Gamma\left(\alpha_{0}-1\right)},
$$

where $c_{0}=I_{t_{0}}^{1-\alpha_{0}} x(0)$ and $c_{1}=I_{t_{0}}^{2-\alpha_{0}} x(0)$. The initial condition $x(0)=0$ implies $c_{1}=0$ which leads to

$$
x(t)=I_{t_{0}}^{\alpha_{0}} f(t, x(t))+c_{0} \frac{t^{\alpha_{0}-1}}{\Gamma\left(\alpha_{0}\right)} .
$$

Using the Riemann-Liouville fractional derivative of order $\alpha_{0}-1$ for (38) on $J_{0}$, we get

$$
D^{\alpha_{0}-1} x(t)=I_{t_{0}}^{1} f(t, x(t))+c_{0} .
$$

From the second initial condition of (5), we get

$$
x(t)=I_{t_{0}}^{\alpha_{0}} f(t, x(t))+\beta \frac{t^{\alpha_{0}-1}}{\Gamma\left(\alpha_{0}\right)} .
$$

Taking the Riemann-Liouville fractional integral of order $1-$ $\alpha_{0}$ and $2-\alpha_{0}$ for (40) and substituting $t=t_{1}$, we have

$$
\begin{aligned}
& I_{t_{0}}^{1-\alpha_{0}} x\left(t_{1}\right)=I_{t_{0}}^{1} f\left(t_{1}, x\left(t_{1}\right)\right)+\beta, \\
& I_{t_{0}}^{2-\alpha_{0}} x\left(t_{1}\right)=I_{t_{0}}^{2} f\left(t_{1}, x\left(t_{1}\right)\right)+\beta t_{1} .
\end{aligned}
$$

For $t \in J_{1}$, taking the Riemann-Liouville fractional integral of order $\alpha_{1}$ for (5), we have

$$
\begin{aligned}
x(t)= & \frac{\left(t-t_{1}\right)^{\alpha_{1}-2}}{\Gamma\left(\alpha_{1}-1\right)} I_{t_{1}}^{2-\alpha_{1}} x\left(t_{1}^{+}\right) \\
& +\frac{\left(t-t_{1}\right)^{\alpha_{1}-1}}{\Gamma\left(\alpha_{1}\right)} I_{t_{1}}^{1-\alpha_{1}} x\left(t_{1}^{+}\right)+I_{t_{1}}^{\alpha_{1}} f(t, x(t)) .
\end{aligned}
$$


Since $I_{t_{1}}^{1-\alpha_{1}} x\left(t_{1}^{+}\right)=I_{t_{0}}^{1-\alpha_{0}} x\left(t_{1}\right)+\varphi_{1}\left(x\left(t_{1}\right)\right)$ and $I_{t_{1}}^{2-\alpha_{1}} x\left(t_{1}^{+}\right)=$ $I_{t_{0}}^{2-\alpha_{0}} x\left(t_{1}\right)+\varphi_{1}^{*}\left(x\left(t_{1}\right)\right)$, it follows that, for $t \in J_{1}$,

$x(t)$

$$
\begin{aligned}
= & \frac{\left(t-t_{1}\right)^{\alpha_{1}-2}}{\Gamma\left(\alpha_{1}-1\right)}\left[\beta t_{1}+I_{t_{0}}^{2} f\left(t_{1}, x\left(t_{1}\right)\right)+\varphi_{1}^{*}\left(x\left(t_{1}\right)\right)\right] \\
& +\frac{\left(t-t_{1}\right)^{\alpha_{1}-1}}{\Gamma\left(\alpha_{1}\right)}\left[\beta+I_{t_{0}}^{1} f\left(t_{1}, x\left(t_{1}\right)\right)+\varphi_{1}\left(x\left(t_{1}\right)\right)\right] \\
& +I_{t_{1}}^{\alpha_{1}} f(t, x(t)) .
\end{aligned}
$$

The Riemann-Liouville integrating of the above equation of order $1-\alpha_{1}$ and $2-\alpha_{1}$ for $t=t_{2}$ leads to

$$
\begin{aligned}
I_{t_{1}}^{2-\alpha_{1}} x\left(t_{2}\right)= & \beta t_{1}+I_{t_{0}}^{2} f\left(t_{1}, x\left(t_{1}\right)\right)+\varphi_{1}^{*}\left(x\left(t_{1}\right)\right) \\
& +\left(t_{2}-t_{1}\right)\left(\beta+I_{t_{0}}^{1} f\left(t_{1}, x\left(t_{1}\right)\right)\right. \\
& +\varphi_{1}\left(x\left(t_{1}\right)\right)+I_{t_{1}}^{2} f\left(t_{2}, x\left(t_{2}\right)\right), \\
I_{t_{1}}^{1-\alpha_{1}} x\left(t_{2}\right)= & \beta+I_{t_{0}}^{1} f\left(t_{1}, x\left(t_{1}\right)\right)+\varphi_{1}\left(x\left(t_{1}\right)\right) \\
& +I_{t_{1}}^{1} f\left(t_{2}, x\left(t_{2}\right)\right) .
\end{aligned}
$$

For $t \in J_{2}$, applying the Riemann-Liouville fractional integral of order $\alpha_{2}$ for (5) and substituting values $I_{t_{2}}^{1-\alpha_{2}} x\left(t_{2}^{+}\right)=$ $I_{t_{1}}^{1-\alpha_{1}} x\left(t_{2}\right)+\varphi_{2}\left(x\left(t_{2}\right)\right)$ and $I_{t_{2}}^{2-\alpha_{2}} x\left(t_{2}^{+}\right)=I_{t_{1}}^{2-\alpha_{1}} x\left(t_{2}\right)+\varphi_{2}^{*}\left(x\left(t_{2}\right)\right)$, we get

$$
\begin{aligned}
& x(t)=\frac{\left(t-t_{2}\right)^{\alpha_{2}-2}}{\Gamma\left(\alpha_{2}-1\right)}\left[\beta t_{2}+\left(t_{2}-t_{1}\right)\left(I_{t_{0}}^{1} f\left(t_{1}, x\left(t_{1}\right)\right)\right.\right. \\
& \left.+\varphi_{1}\left(x\left(t_{1}\right)\right)\right)+\left(I_{t_{0}}^{2} f\left(t_{1}, x\left(t_{1}\right)\right)+\varphi_{1}^{*}\left(x\left(t_{1}\right)\right)\right. \\
& \left.\left.+I_{t_{1}}^{2} f\left(t_{2}, x\left(t_{2}\right)\right)+\varphi_{2}^{*}\left(x\left(t_{2}\right)\right)\right)\right]+\frac{\left(t-t_{2}\right)^{\alpha_{2}-1}}{\Gamma\left(\alpha_{2}\right)}[\beta \\
& +I_{t_{0}}^{1} f\left(t_{1}, x\left(t_{1}\right)\right)+\varphi_{1}\left(x\left(t_{1}\right)\right)+I_{t_{1}} f\left(t_{2}, x\left(t_{2}\right)\right) \\
& \left.+\varphi_{2}\left(x\left(t_{2}\right)\right)\right]+I_{t_{2}}^{\alpha_{2}} f(t, x(t)) .
\end{aligned}
$$

Repeating the above process, for $t \in J$, we obtain (36) as requested.

Next, we will prove the existence and uniqueness of a solution to the initial value problem (5) by using Banach's fixed point theorem.

Theorem 10. Assume that $\left(H_{1}\right)$ and $\left(H_{2}\right)$ hold. In addition we suppose that

$\left(H_{3}\right) \varphi_{k}^{*}: \mathbb{R} \rightarrow \mathbb{R}, k=1,2,3, \ldots, m$, are continuous functions and satisfy

$\left|\varphi_{k}^{*}(x)-\varphi_{k}^{*}(y)\right| \leq L_{3}|x-y|, \quad L_{3}>0, \forall x, y \in \mathbb{R}$.

$$
\begin{aligned}
& \text { If } \\
& \Omega_{1}:=\frac{T_{2}^{*}}{\Gamma_{2}^{*}}\left(L_{1}(\Phi+T+1)+L_{2}(2 m-1)+L_{3} m\right)<1,
\end{aligned}
$$

where

$$
\Phi=\frac{1}{2} \sum_{j=1}^{m}\left(t_{j}-t_{j-1}\right)\left(2 t_{m}-t_{j}-t_{j-1}\right)
$$

$T_{2}^{*}=\max \left\{T^{\gamma+\alpha_{k}-2}, T^{\gamma+\alpha_{k}-1}, T^{\gamma+\alpha_{k}}\right\}, \Gamma_{2}^{*}=\min \left\{\Gamma\left(\alpha_{k}-\right.\right.$ 1), $\Gamma\left(\alpha_{k}\right)$, and $\left.\Gamma\left(\alpha_{k}+1\right)\right\}, \gamma+\alpha_{k}>2$ for $k=$ $0,1,2, \ldots, m$, then problem (5) has a unique solution on $[0, T]$.

Proof. We define the operator $\mathscr{A}: P C(J, \mathbb{R}) \rightarrow P C(J, \mathbb{R})$ as follows:

$$
\begin{aligned}
& \mathscr{A} x(t)=\frac{\left(t-t_{k}\right)^{\alpha_{k}-2}}{\Gamma\left(\alpha_{k}-1\right)}\left[\beta t_{k}\right. \\
& +\sum_{j=1}^{k-1}\left(\left(t_{k}-t_{j}\right)\left(I_{t_{j-1}}^{1} f\left(t_{j}, x\left(t_{j}\right)\right)+\varphi_{j}\left(x\left(t_{j}\right)\right)\right)\right) \\
& \left.+\sum_{j=1}^{k}\left(I_{t_{j-1}}^{2} f\left(t_{j}, x\left(t_{j}\right)\right)+\varphi_{j}^{*}\left(x\left(t_{j}\right)\right)\right)\right] \\
& +\frac{\left(t-t_{k}\right)^{\alpha_{k}-1}}{\Gamma\left(\alpha_{k}\right)}[\beta \\
& \left.+\sum_{j=1}^{k}\left(I_{t_{j-1}}^{1} f\left(t_{j}, x\left(t_{j}\right)\right)+\varphi_{j}\left(x\left(t_{j}\right)\right)\right)\right] \\
& +I_{t_{k}}^{\alpha_{k}} f(t, x(t)),
\end{aligned}
$$

for $t \in J_{k}, k=0,1,2, \ldots, m$ with $\sum_{j=a}^{b}=0$ for $b<a$.

It is straightforward to show that $\mathscr{A} x \in P C_{\gamma}(J, \mathbb{R})$; see Theorem 7. Next we will show that $\mathscr{A} B_{r} \subset B_{r}$, where a ball $B_{r}$ is defined by $B_{r}=\left\{x \in P C_{\gamma}(J, \mathbb{R}),\|x\|_{P C_{\gamma}} \leq r\right\}$. Assume that $\sup _{t \in J}|f(t, 0)|=M, \max \left\{\left|\varphi_{k}(0)\right|: k=1,2,3, \ldots, m\right\}=N$ and $\max \left\{\left|\varphi_{k}^{*}(0)\right|: k=1,2,3, \ldots, m\right\}=P$. Setting

$$
\begin{aligned}
\Omega_{2} & =\frac{T_{2}^{*}}{\Gamma_{2}^{*}}(M(\Phi+T+1)+N(2 m-1)+P m \\
& +|\beta|(T+1))
\end{aligned}
$$

we choose a constant $r$ such that

$$
r \geq \frac{\Omega_{2}}{1-\Omega_{1}} .
$$

Let $x \in B_{r}$. For any $t \in J_{k}, k=0,1,2, \ldots, m$, we have 
Discrete Dynamics in Nature and Society

7

$$
\begin{aligned}
& |(\mathscr{A} x)(t)| \leq \frac{\left(t-t_{k}\right)^{\alpha_{k}-2}}{\Gamma\left(\alpha_{k}-1\right)}\left[|\beta| t_{k}+\sum_{j=1}^{k-1}\left(\left(t_{k}-t_{j}\right)\left(I_{t_{j-1}}^{1}|f(s, x(s))|\left(t_{j}\right)+\left|\varphi_{j}\left(x\left(t_{j}\right)\right)\right|\right)\right)\right. \\
& \left.+\sum_{j=1}^{k}\left(I_{t_{j-1}}^{2}|f(s, x(s))|\left(t_{j}\right)+\left|\varphi_{j}^{*}\left(x\left(t_{j}\right)\right)\right|\right)\right]+\frac{\left(t-t_{k}\right)^{\alpha_{k}-1}}{\Gamma\left(\alpha_{k}\right)}\left[|\beta|+\sum_{j=1}^{k}\left(I_{t_{j-1}}^{1}|f(s, x(s))|\left(t_{j}\right)+\left|\varphi_{j}\left(x\left(t_{j}\right)\right)\right|\right)\right] \\
& +I_{t_{k}}^{\alpha_{k}}|f(s, x(s))|(t) \leq \frac{\left(t-t_{k}\right)^{\alpha_{k}-2}}{\Gamma\left(\alpha_{k}-1\right)}\left[|\beta| t_{k}\right. \\
& +\sum_{j=1}^{k-1}\left(\left(t_{k}-t_{j}\right)\left(I_{t_{j-1}}^{1}(|f(s, x(s))-f(s, 0)|+|f(s, 0)|)\left(t_{j}\right)+\left|\varphi_{j}\left(x\left(t_{j}\right)\right)-\varphi_{j}(0)\right|+\left|\varphi_{j}(x(0))\right|\right)\right) \\
& \left.+\sum_{j=1}^{k}\left(I_{t_{j-1}}^{2}(|f(s, x(s))-f(s, 0)|+|f(s, 0)|)\left(t_{j}\right)+\left|\varphi_{j}^{*}\left(x\left(t_{j}\right)\right)-\varphi_{j}^{*}(0)\right|+\left|\varphi_{j}^{*}(0)\right|\right)\right]+\frac{\left(t-t_{k}\right)^{\alpha_{k}-1}}{\Gamma\left(\alpha_{k}\right)}[|\beta| \\
& \left.+\sum_{j=1}^{k}\left(I_{t_{j-1}}^{1}(|f(s, x(s))-f(s, 0)|+|f(s, 0)|)\left(t_{j}\right)+\left|\varphi_{j}\left(x\left(t_{j}\right)\right)-\varphi_{j}(0)\right|+\left|\varphi_{j}(0)\right|\right)\right]+I_{t_{k}}^{\alpha_{k}}(|f(s, x(s))-f(s, 0)| \\
& +|f(s, 0)|)(t) \leq \frac{\left(t-t_{k}\right)^{\alpha_{k}-2}}{\Gamma\left(\alpha_{k}-1\right)}\left[|\beta| t_{k}+\left(L_{1} r+M\right)\left(\sum_{j=1}^{k-1}\left(t_{k}-t_{j}\right)\left(t_{j}-t_{j-1}\right)\right)+\left(L_{2} r+N\right)(k-1)\right. \\
& \left.+\left(L_{1} r+M\right)\left(\sum_{j=1}^{k} \frac{\left(t_{j}-t_{j-1}\right)^{2}}{2}\right)+\left(L_{3} r+P\right) k\right]+\frac{\left(t-t_{k}\right)^{\alpha_{k}-1}}{\Gamma\left(\alpha_{k}\right)}\left[|\beta|+\left(L_{1} r+M\right)\left(\sum_{j=1}^{k}\left(t_{j}-t_{j-1}\right)\right)+\left(L_{2} r+N\right) k\right] \\
& +\left(L_{1} r+M\right) \frac{\left(t-t_{k}\right)^{\alpha_{k}}}{\Gamma\left(\alpha_{k}+1\right)} .
\end{aligned}
$$

Multiplying both sides of the above inequality by $\left(t-t_{k}\right)^{\gamma}$ for $t \in J_{k}$, we have

$$
\begin{array}{ll}
\left(t-t_{k}\right)^{\gamma}|(\mathscr{A} x)(t)| \leq \frac{\left(t-t_{k}\right)^{\gamma+\alpha_{k}-2}}{\Gamma\left(\alpha_{k}-1\right)}\left[|\beta| t_{k}\right. & +\left(L_{1} r+M\right) \frac{\left(t-t_{k}\right)^{\gamma+\alpha_{k}}}{\Gamma\left(\alpha_{k}+1\right)} \leq \frac{T_{2}^{*}}{\Gamma_{2}^{*}}[|\beta| T \\
+\left(L_{1} r+M\right)\left(\sum_{j=1}^{k-1}\left(t_{k}-t_{j}\right)\left(t_{j}-t_{j-1}\right)\right) & +\left(L_{1} r+M\right)\left(\sum_{j=1}^{m-1}\left(t_{m}-t_{j}\right)\left(t_{j}-t_{j-1}\right)\right) \\
+\left(L_{2} r+N\right)(k-1) & +\left(L_{2} r+N\right)(m-1) \\
\left.+\left(L_{1} r+M\right)\left(\sum_{j=1}^{k} \frac{\left(t_{j}-t_{j-1}\right)^{2}}{2}\right)+\left(L_{3} r+P\right) k\right] & +\left(L_{1} r+M\right)\left(\sum_{j=1}^{m} \frac{\left(t_{j}-t_{j-1}\right)^{2}}{2}\right)+\left(L_{3} r+P\right)+|\beta| \\
+\frac{\left(t-t_{k}\right)^{\gamma+\alpha_{k}-1}}{\Gamma\left(\alpha_{k}\right)}[|\beta| & +\left(L_{1} r+M\right)\left(\sum_{j=1}^{m}\left(t_{j}-t_{j-1}\right)\right)+\left(L_{2} r+N\right) m
\end{array}
$$

$\left.+\left(L_{1} r+M\right)\left(\sum_{j=1}^{k}\left(t_{j}-t_{j-1}\right)\right)+\left(L_{2} r+N\right) k\right]$ 


$$
\begin{aligned}
& \left.+\left(L_{1} r+M\right)\right] \\
& =r\left[\frac{T_{2}^{*}}{\Gamma_{2}^{*}}\left(L_{1}(\Phi+T+1)+L_{2}(2 m-1)+L_{3} m\right)\right]
\end{aligned}
$$

$$
\begin{aligned}
& +\frac{T_{2}^{*}}{\Gamma_{2}^{*}}(M(\Phi+T+1)+N(2 m-1)+P m \\
& +|\beta|(T+1))=\Omega_{1} r+\Omega_{2} .
\end{aligned}
$$

This implies that $\mathscr{A} B_{r} \subset B_{r}$.

Finally we will show that $\mathscr{A}$ is a contraction mapping on $B_{r}$. For $x, y \in P C_{\gamma}(J, \mathbb{R})$ and for each $t \in J$ we have

$$
\begin{aligned}
& |(\mathscr{A} x)(t)-(\mathscr{A} y)(t)| \leq \frac{\left(t-t_{k}\right)^{\alpha_{k}-2}}{\Gamma\left(\alpha_{k}-1\right)}\left[\sum_{j=1}^{k-1}\left(\left(t_{k}-t_{j}\right)\left(I_{t_{j-1}}^{1}|f(s, x(s))-f(s, y(s))|\left(t_{j}\right)+\left|\varphi_{j}\left(x\left(t_{j}\right)\right)-\varphi_{j}\left(y\left(t_{j}\right)\right)\right|\right)\right)\right. \\
& \left.\quad+\sum_{j=1}^{k}\left(I_{t_{j-1}}^{2}|f(s, x(s))-f(s, y(s))|\left(t_{j}\right)+\left|\varphi_{j}^{*}\left(x\left(t_{j}\right)\right)-\varphi_{j}^{*}\left(y\left(t_{j}\right)\right)\right|\right)\right] \\
& \quad+\frac{\left(t-t_{k}\right)^{\alpha_{k}-1}}{\Gamma\left(\alpha_{k}\right)}\left[\sum_{j=1}^{k}\left(I_{t_{j-1}}^{1}|f(s, x(s))-f(s, y(s))|\left(t_{j}\right)+\left|\varphi_{j}\left(x\left(t_{j}\right)\right)-\varphi_{j}\left(y\left(t_{j}\right)\right)\right|\right)\right]+I_{t_{k}}^{\alpha_{k}}|f(s, x(s))-f(s, y(s))|
\end{aligned}
$$

$\cdot(t)$

Multiplying both sides of the above inequality by $\left(t-t_{k}\right)^{\gamma}$, we have

$$
\begin{aligned}
& \left|\left(t-t_{k}\right)^{\gamma}(\mathscr{A} x)(t)-\left(t-t_{k}\right)^{\gamma}(\mathscr{A} y)(t)\right| \\
& \leq \frac{\left(t-t_{k}\right)^{\gamma+\alpha_{k}-2}}{\Gamma\left(\alpha_{k}-1\right)}\left[L_{1}\|x-y\|\right. \\
& \cdot\left(\sum_{j=1}^{k-1}\left(t_{k}-t_{j}\right)\left(t_{j}-t_{j-1}\right)\right)+L_{2}\|x-y\|(k-1) \\
& \left.+L_{1}\|x-y\|\left(\sum_{j=1}^{k} \frac{\left(t_{j}-t_{j-1}\right)^{2}}{2}\right)+L_{3}\|x-y\| k\right] \\
& +\frac{\left(t-t_{k}\right)^{\gamma+\alpha_{k}-1}}{\Gamma\left(\alpha_{k}\right)}\left[L_{1}\|x-y\|\left(\sum_{j=1}^{k}\left(t_{j}-t_{j-1}\right)\right)\right. \\
& \left.+L_{2}\|x-y\| k\right]+L_{1}\|x-y\| \frac{\left(t-t_{k}\right)^{\gamma+\alpha_{k}}}{\Gamma\left(\alpha_{k}+1\right)} \\
& \leq \Omega_{1}\|x-y\| .
\end{aligned}
$$

It follows that

$$
\|\mathscr{A} x-\mathscr{A} y\| \leq \Omega_{1}\|x-y\|
$$

Since $\Omega_{1}<1, \mathscr{A}$ is a contraction mapping on $B_{r}$. Therefore (5) has a unique solution on $[0, T]$.
Example 11. Consider the following impulsive multiorders Riemann-Liouville fractional initial value problem:

$$
\begin{aligned}
D_{t_{k}}^{\left(2 k^{2}+3 k+5\right) /\left(k^{2}+2 k+3\right)} x= & \frac{2^{t}|x(t)| \cos t}{(7+|x(t)|)^{2}+|x(t)| \sin ^{2} t} \\
& +\frac{1}{2}, \\
t \in\left[0, \tan ^{-1}\left(\frac{11}{3 \pi}\right)\right], t \neq t_{k}, & \\
\tilde{\Delta} x\left(t_{k}\right)= & \frac{\left|x\left(t_{k}\right)\right|}{9\left(k^{2}+3 k+5\right)+\left|x\left(t_{k}\right)\right|}, \\
k= & 1,2, \ldots, 10, t_{k}=\tan ^{-1}\left(\frac{k}{3 \pi}\right), \\
\Delta^{*} x\left(t_{k}\right) & =\frac{\left|x\left(t_{k}\right)\right|}{37(1-2 \cos k \pi)+\left|x\left(t_{k}\right)\right|}, \\
k & =1,2, \ldots, 10, t_{k}=\tan ^{-1}\left(\frac{k}{3 \pi}\right), \\
x(0) & =0 \\
D_{t_{0}}^{2 / 3} x(0) & =e .
\end{aligned}
$$

Here $\alpha_{k}=\left(2 k^{2}+3 k+5\right) /\left(k^{2}+2 k+3\right), k=0,1,2, \ldots, 10$, $m=10, T=\tan ^{-1}(11 /(3 \pi)), f(t, x)=\left(2^{t}|x| \cos t\right) /\left((7+|x|)^{2}+\right.$ $\left.|x| \sin ^{2} t\right)+(1 / 2), \varphi_{k}(x)=|x| /\left(9\left(k^{2}+3 k+5\right)+|x|\right)$, and $\varphi_{k}^{*}(x)=$ $|x| /(37(1-2 \cos k \pi)+|x|)$. Since $|f(t, x)-f(t, y)| \leq(2 / 49) \mid x-$ $y|,| \varphi_{k}(x)-\varphi_{k}(y)|\leq(1 / 81)| x-y \mid$, and $\left|\varphi_{k}^{*}(x)-\varphi_{k}^{*}(y)\right| \leq$ $(3 / 37)|x-y|$, then $\left(H_{1}\right),\left(H_{2}\right)$, and $\left(H_{3}\right)$ are satisfied with $L_{1}=$ $2 / 49, L_{2}=1 / 81$, and $L_{3}=3 / 37$, respectively. By choosing 
$\gamma=2$, we can find that $T_{2}^{*}=0.781307, \Gamma_{2}^{*}=0.902745, \Phi=$ 0.332114 , and

$$
\begin{aligned}
\Omega_{1} & =\frac{T_{2}^{*}}{\Gamma_{2}^{*}}\left(L_{1}(\phi+T+1)+L_{2}(2 m-1)+L_{3} m\right) \\
& =0.982275<1 .
\end{aligned}
$$

Hence, by Theorem 10, the initial value problem (57) has a unique solution on $\left[0, \tan ^{-1} 11 /(3 \pi)\right]$.

\section{Conflict of Interests}

The authors declare that there is no conflict of interests regarding the publication of this paper.

\section{Acknowledgment}

This research is supported by the Centre of Excellence in Mathematics, the Commission on Higher Education, Thailand.

\section{References}

[1] R. P. Agarwal, Y. Zhou, and Y. He, "Existence of fractional neutral functional differential equations," Computers \& Mathematics with Applications, vol. 59, no. 3, pp. 1095-1100, 2010.

[2] B. Ahmad and J. J. Nieto, "Boundary value problems for a class of sequential integrodifferential equations of fractional order," Journal of Function Spaces and Applications, vol. 2013, Article ID 149659, 8 pages, 2013.

[3] B. Ahmad and S. K. Ntouyas, "Fractional differential inclusions with fractional separated boundary conditions," Fractional Calculus and Applied Analysis, vol. 15, no. 3, pp. 362-382, 2012.

[4] D. Baleanu, K. Diethelm, E. Scalas, and J. J. Trujillo, Fractional Calculus: Models and Numerical Methods, Series on Complexity, Nonlinearity and Chaos, World Scientific Publishers, Boston, Mass, USA, 2012.

[5] A. A. Kilbas, H. M. Srivastava, and J. J. Trujillo, Theory and Applications of Fractional Differential Equations, vol. 204 of North-Holland Mathematics Studies, Elsevier Science B.V., Amsterdam, The Netherlands, 2006.

[6] K. S. Miller and B. Ross, An Introduction to the Fractional Calculus and Fractional Differential Equations, John Wiley \& Sons, New York, NY, USA, 1993.

[7] I. Podlubny, Fractional Differential Equations, Academic Press, San Diego, Calif, USA, 1999.

[8] G. Wang, S. Liu, D. Baleanu, and L. Zhang, "A new impulsive multi-orders fractional differential equation involving multipoint fractional integral boundary conditions," Abstract and Applied Analysis, vol. 2014, Article ID 932747, 10 pages, 2014.

[9] C. Bai, "Impulsive periodic boundary value problems for fractional differential equation involving Riemann-Liouville sequential fractional derivative," Journal of Mathematical Analysis and Applications, vol. 384, no. 2, pp. 211-231, 2011.

[10] M. Fečkan, Y. Zhou, and J. Wang, "On the concept and existence of solution for impulsive fractional differential equations,"
Communications in Nonlinear Science and Numerical Simulation, vol. 17, no. 7, pp. 3050-3060, 2012.

[11] X. Zhang, X. Zhang, and M. Zhang, "On the concept of general solution for impulsive differential equations of fractional order $q \in(0,1), "$ Applied Mathematics and Computation, vol. 247, pp. $72-89,2014$. 


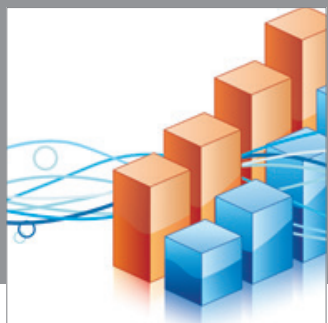

Advances in

Operations Research

mansans

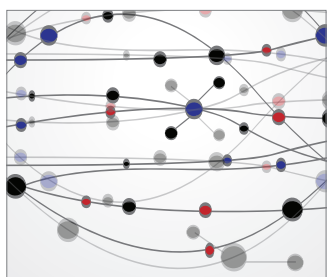

The Scientific World Journal
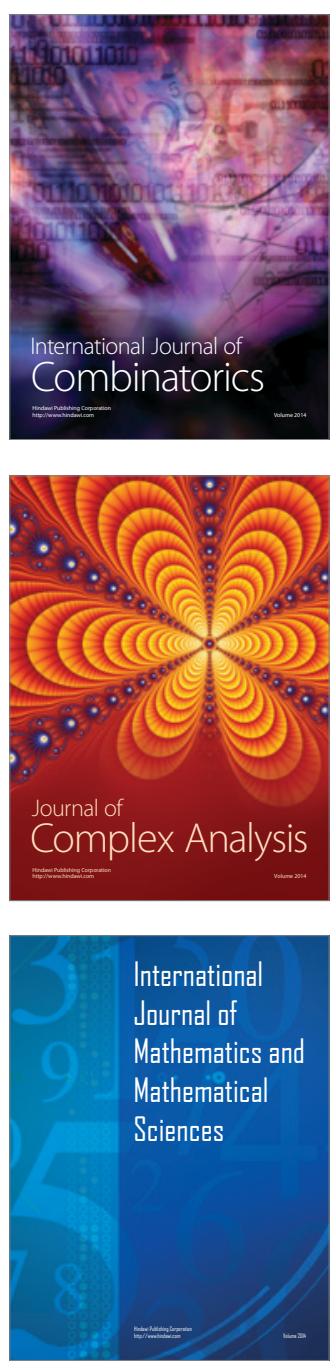
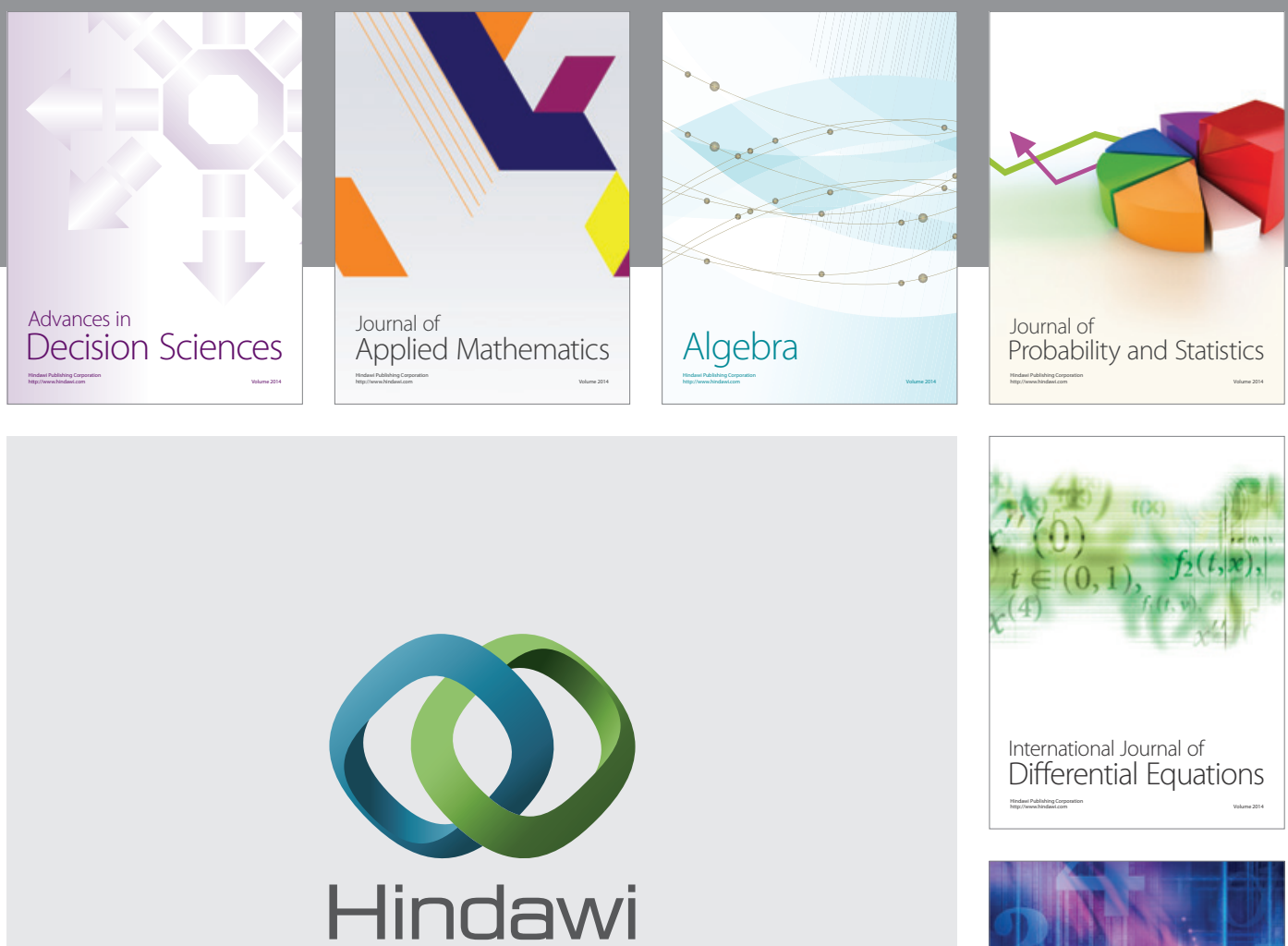

Submit your manuscripts at http://www.hindawi.com
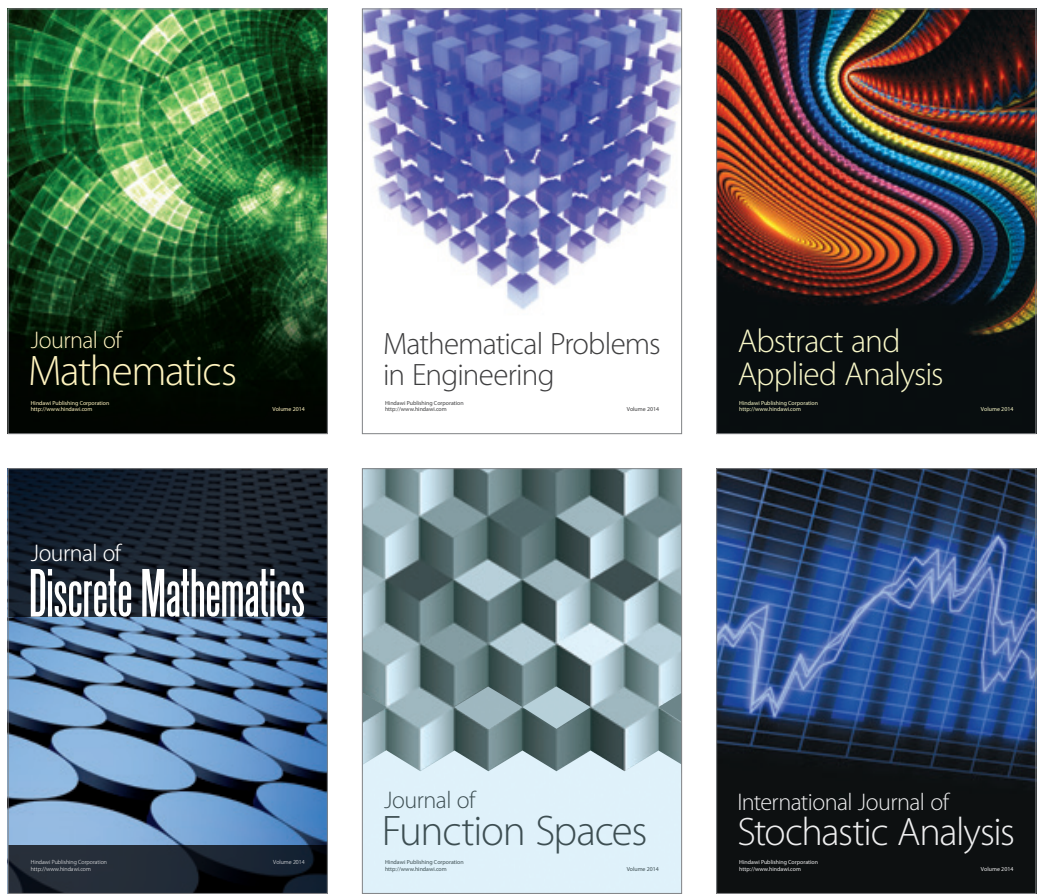

Journal of

Function Spaces

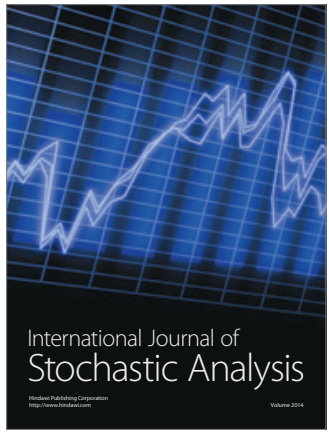

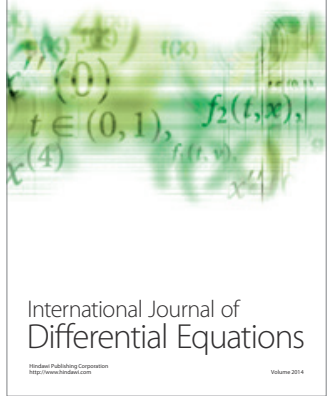
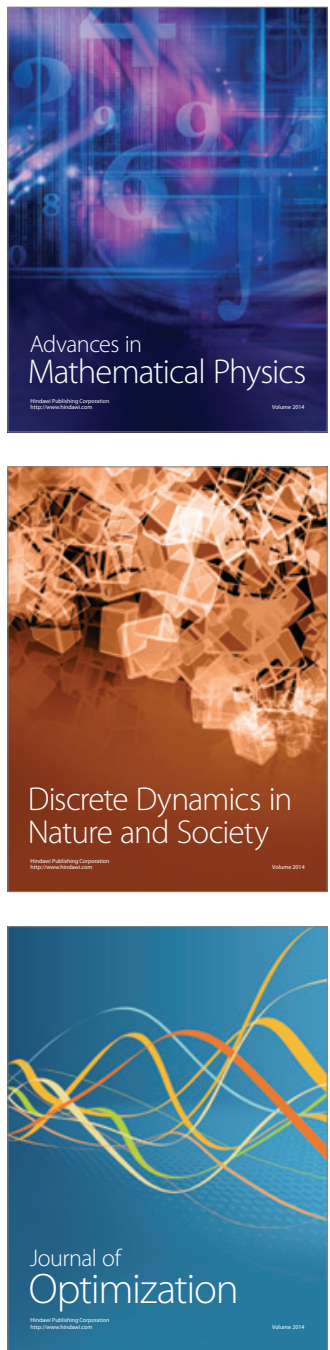